\section{Demonstration für die Phytotherapie - in Baden müssen alle dabei sein}

Zum 19. Mal findet am 18. November 2004 die Schweizerische Jahrestagung für Phytotherapie statt. Seit es diese Tagung gibt, steht sie im Zeichen der Evidenz: Die Veranstaltungen wollen zeigen, dass die phytotherepeutische Tradition wissenschaftlich untermauert werden kann und in vielen Fällen wissenschaftlich untermauert ist. Bei der diesjährigen Tagung steht die Evidenz als Thema im Mittelpunkt.

Seit mehreren Jahren beschäftigt das Projekt Evaluation Komplementärmedizin die Schweizerische Medizinische Gesellschaft für Phytotherapie. Dieses Projekt wurde lanciert, um Wirksamkeit, Zweckmässigkeit und Wirtschaftlichkeit von fünf der Komplementärmedizin zugerechneten Methoden zu beurteilen. Diese wurden mit der Einführung des neuen Krankenversicherungsgesetzes befristet in den Leistungskatalog der Grundversicherung aufgenommen. Dazu zählt auch die Phytotherapie. Zahlreiche Kolleginnen und Kollegen haben mitgearbeitet und sich engagiert. Die Zeit ist reif, sich mit den Resultaten zu beschäftigen. Noch sind die Ergebnisse der Studien im Detail nicht bekannt. Bekannt ist jedoch, dass mit der Phytotherapie positive Erfahrungen gemacht werden und dass die wissenschaftliche Aufarbeitung der Daten auch ausserhalb des Projektes wahrgenommen wird. Drei Referenten werden diese Sicht der Dinge einbringen. Impulsreferate zum Abschluss der Tagung sollen Wege aufzeigen, wie die SMGP im nächsten Jahr mit den Resultaten umgehen soll und welche Anträge bezüglich Grundversicherung gestellt werden sollen. Dr. med. H.H. Brunner, seit wenigen Wochen Vizedirektor Kranken- und Unfallversicherungen beim Bundesamt für Gesundheit, hat für diesen Teil sein Kommen zugesagt.

Die Tagung hat eine ausgeprägte gesundheitspolitische Dimension. Die SMGP ist gemäss Statuten verpflichtet, für den Erhalt der Phytotherapie im Schweizerischen Gesundheitswesen zu kämpfen. In Zeiten der Unsicherheit ist dieser Einsatz von besonderer Bedeutung. Mithelfen kann jedermann, der ein Interesse an der Phytotherapie hat. Mit der Teilnahme an dieser Tagung soll und muss dieses Engagement dokumentiert werden. Ein volles Haus setzt klare Zeichen: nämlich ein breites Interesse an der Phytotherapie in Medizin und Pharmazie. Entsprechend müsste es für alle an Phytotherapie interessierten Medizinalpersonen und Wissenschafter Ehrensache sein, an dieser Tagung teilzunehmen!
Zur diesjährigen Jahrestagung passt, dass die SMGP im Herbst mit einem neuen Ausbildungszyklus begonnen hat. Das Programm wurde in diesem Jahr, aufbauend auf den bisherigen Erfahrungen, neu definiert und umfasst nun 11 Kurse sowie den 3-tägigen Grundkurs und kann innerhalb von 3 Jahren absolviert werden. Die Ausbildung führt zum Zertifikat Phytotherapie. Obwohl der Zyklus einem modularen Aufbau folgt, ist derzeit ein Einstieg besonders günstig.

Die SMGP ist zu Recht stolz darauf, eine in dieser Art in Europa einmalige Ausbildung anbieten zu können. Wir sind stolz, aus einem Brief von PD Dr. med. Jakob Eberhard, Chefarzt an der Frauenklinik des Kantonsspitals Frauenfeld, folgende Worte zitieren zu dürfen: «Es war für mich eine grosse Bereicherung an einem Fortbildungskurs Ihrer Gesellschaft mitzumachen und vor allem auch zuhören zu können. Ich habe sehr viele Tipps für unsere urogynäkologische Sprechstunde erfahren und ich hoffe, dass wir damit vielen Patientinnen eine ergänzende Hilfe anbieten können.» Ein Einstieg ist auch im kommenden Jahr möglich, z.B. am 27. Januar zum Thema Herz/Kreislauf.

Beat Meier, Präsident SMGP

Weitere Infos, Programme, Links, Anmeldungsformulare unter www.smgp.ch. Infos zum PEK und dessen Zielen sowie erste Ergebnisse unter www.pekswiss.ch.

\section{Aufruf}

Im Anschluss an die Tagung findet die jährliche Generalversammlung der SMGP statt. Die SMGP steht vor einer umfassenden Strukturänderung: Neu wird eine Geschäftsstelle eingerichtet; die Weiterbildung wird nach dem Vorbild der Romands regionalisiert und von zwei Regionalkomitees (Romandie und Deutschschweiz) organisiert. Mit der neuen Struktur möchte der Vorstand die Mitglieder aus der Romandie stärker in die SMGP einbinden, die Zweisprachigkeit fördern und die Schlagkraft der Gesellschaft vergrössern. Die neue Struktur mit entsprechenden Änderungen der Statuten muss von der Generalversammlung genehmigt werden. Auch steht eine Neuwahl des Vorstands an. Der Vorstand bittet daher alle Kolleginnen und Kollegen, die Jahresversammlung zu besuchen und mit ihrer Teilnahme die Reform zu unterstützen. 


\section{Schweizerische Tagung für Phytotherapie: Evaluation Phytotherapie - eine Bilanz}

18. November 20004, Kongresszentrum TRAFO, Baden AG

Themen

Nutzen - Risiko - Kosten am Beispiel von Ginkgo biloba

Phytotherapie in den Schweizer Arztpraxen: PEK-Studie

Phytotherapie und konventionelle Medizin: vergleichende Analyse

Klinische Studien in der Phytotherapie

Evidenz Phytotherapie aus der Sicht eines Hochschulmediziners

Health Technology Assessment Report

Phytotherapie und Grundversicherung: Strategie für die Zukunft

\section{Anmeldung}

Sekretariat SMGP c/o Frau Bacs

Keltenstr. 43

CH-8054 Zürich

Tel. +41 1 252-18 79, Fax -19 06

E-mail sekretariat-smgp@ swissonline.ch

\section{SMGP trotzt dem Regen auf der Exkursion im Maderanertal}

Das sprichwörtliche Wetterglück verliess die Exkursionsfreunde der SMGP für einmal: Ausgerechnet am Donnerstag, den 26. August, regnete es im Maderanertal in Strömen. Die Pflanzen kümmerten sich wenig darum, im Gegenteil: Anstelle der Sonne leuchteten grosse Bestände von Solidago virgaurea (gewöhnliche Goldrute) den Exkursionsteilnehmern entgegen, und auch die Bestände von Origanum vulgare (Dost) liessen an blau-violetten Farbnuancen - und natürlich am Geruchsbouquet - nichts zu wünschen übrig. Die Goldrute, eines der effizientesten Aquaretika der Phytotherapie, steht häufig neben verschiedenen Senecio-Arten. Eine gewisse Verwechslungsgefahr besteht. Die einreihige Anordnung der Kelchblätter rund um die Blüte von Solidago virgaurea unterscheidet sich aber eindeutig von der dachziegelartigen Anordnung bei den toxischen Kreuzkräutern (Senecio-Spezies).

Nässe und Kälte konnten die Exkursion nicht zum Halten bringen, zumal der Wirt des Bergrefugiums beim Golzerensee seinen kleinen Pavillon einheizte und mit eigenen Kräutern eine phytotherapeutische «Gemüsesuppe» in die Teller zauberte. Der Ausbildungsteil wurde kurzerhand in den Pavillon verlegt: Botaniker Adrian Leuchmann und Mediziner Peter Kälin hatten die Pflanzen gesammelt und beleuchteten nun botanische und medizinische Aspekte. Am Nachmittag ging es dann noch einmal ins Freie: Studiert wurde die Moorflora mit den Fleisch fressenden Pflanzen, insbesondere Drosera rotundifolia, den Ericaceen, namentlich Vaccinium uliginosum und Vaccinium myrtillus (Moor- und Heidelbeere) sowie verblühten und noch blühenden Orchideen. Der Rückweg erlaubte einen Blick auf die wunderschönen Bestände von Menyanthes trifoliata (Fieberklee) an den Ufern des idyllischen Golzerensees.

Die botanische Besonderheit dieser Exkursion wuchs auf einer Arzneipflanze, nämlich der Brennnessel. Die Brennnesseln litten sichtlich unter dem Cuscuta europaea genannten Schmarotzer. Am Schluss waren sich die mehr als zwanzig Exkursionsteilnehmer einig: Es ist richtig, Exkursionen bei jedem Wetter durchzuführen. 